\section{Complement system on the attack in autoimmunity}

\author{
John P. Atkinson
}

Washington University School of Medicine, St. Louis, Missouri, USA

\begin{abstract}
The antiphospholipid syndrome is characterized clinically by fetal loss and thrombosis and serologically by the presence of autoantibodies to lipid-binding proteins. In a model of this procoagulant condition in which these antibodies are injected into pregnant mice, fetal loss was prevented by blocking of complement activation. Specifically, interaction of complement component $5 \mathrm{a}$ (C5a) with its receptor is necessary for thrombosis of placental vasculature (see the related article beginning on page 1644). Inhibition of complement activation may have a therapeutic role in this disease.
\end{abstract}

J. Clin. Invest. 112:1639-1641 (2003). doi:10.1172/JCI200320309.

In the first autoimmune condition that was recognized as such over a century ago, antibody and complement were shown to lyse human red blood cells in a rare form of hemolytic anemia known as paroxysmal cold hemoglobinuria (1). Impressively, these same two factors had been found to mediate lysis of bacterial pathogens just three years earlier in 1892. While lysis was the end point in those early studies because it was both pathologically meaningful and easy to monitor, we now realize that it is the phlogistic and opsonic activities of complement that account for much of its physiologic and pathologic importance. The antiphospholipid syndrome (APS) is an autoimmune disease in which autoantibodies are synthesized to lipids and lipid-binding proteins. These antibodies bind to antigens expressed by endothelial cells and produce a clinical syndrome featuring thrombosis. Fetal wastage is caused by placental infarction. In this issue of the JCI, Girardi et al. report that activation of the complement

Address correspondence to: John P.

Atkinson, Campus Box 8045, 660 South

Euclid Avenue, St. Louis, Missouri

63110-1093, USA. Phone: (314) 362-8391;

Fax: (314) 362-1366;

E-mail: jatkinso@im.wustl.edu.

Conflict of interest: The author has declared

that no conflict of interest exists.

Nonstandard abbreviations used: antiphospholipid syndrome (APS); alternative pathway (AP); complement component 5a (C5a); rheumatoid arthritis (RA). cascade by the immune complexes is required for disease development in a mouse model of APS (2).

Two informative models of humoral autoimmunity that require complement activation to mediate tissue injury

Rheumatoid arthritis. In one model, that of a spontaneously arising inflammatory arthritis, autoantibodies reactive to the ubiquitously expressed intracellular protein glucose-6-phosphate isomerase are synthesized (3-5). These IgG autoantibodies bind to this autoantigen in the joint and trigger a destructive arthritis. Additionally, an acute transient form of synovitis can be produced by the passive transfer of antibody alone. While antibody-mediated activa-
Figure 1 plement activation on self tissue. tion of the classical pathway is a timehonored concept, antibody can also serve as a site that is protected from plasma and membrane inhibitors of complement activation. In particular, these regulators of complement activation are designed to block amplification of the alternative pathway (AP) on self tissue (6). To produce arthritis, antibody binding at a site relatively deficient in complement inhibitors allows "firing" of the AP (5). Complement component $5 \mathrm{a}(\mathrm{C} 5 \mathrm{a})$, interacting with its G-coupled receptor, is absolutely required to recruit neutrophils and to activate nearby cells (5). Based on depletion studies and knockouts, neutrophils, mast cells, and $\mathrm{Fc}$ receptors are also necessary in this model of rheumatoid arthritis (RA) $(5,7)$.

Antiphospholipid syndrome. In the second mouse model of autoimmunity, antiphospholipid antibodies derived from patients are injected into pregnant mice. Remarkably, these antibodies react with lipid-binding proteins on endothelial cells to trigger complement activation, thrombosis, and fetal death secondary to placental insufficiency (8-11). These pathologic antibodies attach to lipid-rich antigens on the developing placental vasculature and then bind complement (12-15). The complement system in turn damages the endothelial cells, leading to a procoagulant state and undesirable thrombosis. In this issue of the JCI, Girardi et al. (2) convincingly demonstrate that both the classical and alternative pathways are

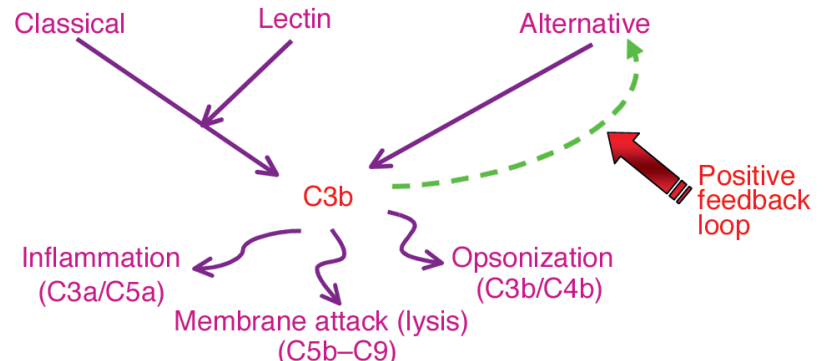

C3b can be deposited on a target by immune complexes that commonly engage the classical pathway, by lectins such as mannose-binding protein that bind to sugars, or through the continuous low-grade turnover of the AP. The deposited C3b serves as a nidus for amplification of $\mathrm{C} 3 \mathrm{~b}$ deposition via the positive feedback loop. Control of this amplification process is critical to prevent undesirable injury to host cells. The presence of antibodies or lectins on a membrane at a site relatively lacking in membrane regulators is conducive to excessive com- 
Requirements for complement activation on self tissue in autoimmunity

Autoantibodies

Protected site relative to complement inhibitors

Antibodies themselves are a protected site. If they also bind to antigen located in a site that is relatively deficient in regulators (such as joint cartilage in the RA model or developing placental vasculature in the APS model), a particularly tissue-damaging situation occurs.

required to generate sufficient $\mathrm{C} 5$ cleavage to cause fetal loss. The released $\mathrm{C} 5 \mathrm{a}$ binds to its receptor on neutrophils and endothelial cells. In this model, neutrophils are again required but $F c \gamma$ receptors are not. Simply put, a procoagulant state is set up, clots form, placental infarction ensues, and embryos die.

\section{Local requirements for complement activation}

The complement-activation scheme is different in the two models. In the APS model, the classical pathway triggers the process by depositing a relatively small amount of $\mathrm{C} 3 \mathrm{~b}$ on the target, and then this $\mathrm{C} 3 \mathrm{~b}$ serves as a nidus for the AP's self-amplifying feedback loop (Figure 1). The AP deposits the majority of the $\mathrm{C} 3 \mathrm{~b}$ and cleaves most of the C5. Another particularly intriguing aspect of this model is the indication that neutrophils enhance the complement-activation process. In the RA model, the AP alone can provide the necessary $\mathrm{C} 3 \mathrm{~b}$ and subsequent $\mathrm{C} 5$ activation (5). As noted, plasma and membrane complement regulators are particularly effective at blocking the feedback loop of the AP $(16,17)$ but are less able to control antibody-mediated classical-pathway activation. Thus, this potentially lethal amplification process occurs routinely and robustly against foreign materials (which in most cases lack regulators). This line of reasoning accounts for why large amounts of autoantibodies that activate the classical pathway are so damaging, yet low levels of autoantibodies or autoreactive lectins are so well tolerated.

Consequently, for excessive and unwanted complement activation to occur on self tissue, something is likely to be amiss relative to the host's complement inhibitors (see Requirements for complement activation on selftissue in autoimmunity). This point is clearly illustrated in the arthritis model, where the evidence points to antibody binding and complement activation taking place at a site relatively deficient in regulators (5). Furthermore, the autoantibodies themselves serve as a protected site for complement activation (6). Genetic deficiencies of complement inhibitors reinforce these points. For example, in paroxysmal nocturnal hemoglobinuria, red blood cells are lysed because of an acquired clonal stem cell inactivation of the enzyme required to anchor two regulators of complement on the erythrocyte membrane (18). In atypical (nondiarrheal) hemolytic uremic syndrome, heterozygous deficiency of one of the complement inhibitors factor $\mathrm{H}$ or CD46 predisposes to a thrombotic microangiopathy because of excessive complement activation $(17,19)$. The take-home lessons from these reports are that complement activation on vessel walls must be rigorously controlled and that even heterozygous deficiency of an inhibitor predisposes to thrombosis if a vessel wall is under siege. A reasonable hypothesis, therefore, to explain clot formation in APS is that the developing placental vasculature is relatively deficient in complementinhibitor function.

\section{Implications for therapy}

Are these pathologic situations in the mouse similar to the human diseases RA and APS? At the minimum, these studies identify signaling pathways and mechanisms associated with tissue destruction that should be further investigated in individuals suffering from RA or APS. At the maximum, they represent almost exactly what takes place in RA and APS. In the former case, one would argue that we have yet to define the autoantibody and autoantigen combination. In the latter case, one would say that the same pathologic process occurs in the human placenta and on other vascular endothelial cells to cause venous and arterial thrombosis.

What, then, are the implications for the treatment of these conditions with a complement inhibitor? The trial of an $\mathrm{mAb}$ that inhibits $\mathrm{C} 5$ was efficacious in RA but not to the same extent as inhibition of TNF (20). For APS, treatment has focused primarily on anticoagulation (21). Complement inhibition would seem to be an attractive therapeutic target in some of these patients. In particular, $\mathrm{C} 5 \mathrm{a}$ and its receptor should now become prime targets for inhibition. Girardi and colleagues have substantially advanced our understanding of the APS model by showing us factors required to mediate the associated tissue injury, and this, in turn, may have therapeutic implications (2).

1. Silverstein, A.M. 1989. A bistory of immunology Academic Press Inc. San Diego, California, USA 422 pp.

2. Girardi, G., et al. 2003. Complement C5a receptors and neutrophils mediate fetal injury in the antiphospholipid syndrome. J. Clin. Invest. 112:1644-1654. doi:10.1172/JCI200318817.

3. Matsumoto, I., Staub, A., Benoist, C., and Mathis, D. 1999. Arthritis provoked by linked T and B cell recognition of a glycolytic enzyme. Science 286:1732-1735.

4. Matsumoto, I., et al. 2002. How antibodies to a ubiquitous cytoplasmic enzyme may provoke joint-specific autoimmune disease. Nat. Immunol. 3:360-365.

5. Ji, H., et al. 2002. Arthritis critically dependent on innate immune system players. Immunity. 16:157-168.

6. Ratnoff, W.D., Fearon, D.T., and Austen, K.F. 1983. The role of antibody in the activation of the alternative complement pathway. Springer Semin Immunopathol. 6:361-371.

7. Wipke, B.T., and Allen, P.M. 2001. Essential role of neutrophils in the initiation and progression of a murine model of rheumatoid arthritis J. Immunol. 167:1601-1608.

8. Branch, D.W., et al. 1990. Immunoglobulin G fractions from patients with antiphospholipid antibodies cause fetal death in BALB/c mice: a model for autoimmune fetal loss. Am. J. Obstet. Gynecol. 163:210-216.

9. Blank, M., Cohen, J., Toder, V., and Shoenfeld, Y. 1991. Induction of antiphospholipid syndrome in naive mice with mouse lupus monoclonal and human polyclonal antibodies. Proc. Natl. Acad. Sci. U. S. A. 88:3069-3073.

10. Piona, A., et al. 1995. Placental thrombosis and fetal loss after passive transfer of mouse lupus monoclonal or human polyclonal anti-cardiolipin antibodies in pregnant naive BALB/c mice. Scand. J. Immunol. 41:427-432.

11. Ikematsu, W., et al. 1998. Human anticardiolipin monoclonal autoantibodies cause placenta necrosis and fetal loss in BALB/c mice. Arthriti Rheum. 41:1026-1039.

12. Rand, J.H., et al. 1997. Pregnancy loss in the antiphospholipid-antibody syndrome: a possible thrombogenic mechanism. N. Engl. J. Med. 337:154-160.

13. Pierangeli, S.S., et al. 1999. Antiphospholipid antibodies from antiphospholipid syndrome patients activate endothelial cells in vitro and in 
vivo Circulation 99:1997-2002.

14. Simantov, R., et al. 1995. Activation of cultured vascular endothelium by antiphospholipid antibodies. J. Clin. Invest. 96:2211-2219.

15. Laudes, I.J., et al. 2002. Expression and function of $\mathrm{C} 5$ a receptor in mouse microvascular endothelial cells. J. Immunol. 169:5962-5970. 16. Barilla-LaBarca, M.L., Liszewski, M.K., Lambris, J.D., Hourcade, D., and Atkinson, J.P. 2002. Role of membrane cofactor protein (CD46) in regulation of $\mathrm{C} 4 \mathrm{~b}$ and $\mathrm{C} 3 \mathrm{~b}$ deposited on cells.
J. Immunol. 168:6298-6304.

17. Manuelian, T., et al. 2003. Mutations in factor $\mathrm{H}$ reduce binding affinity to $\mathrm{C} 3 \mathrm{~b}$ and heparin and surface attachment to endothelial cells in hemolytic uremic syndrome. J. Clin. Invest. 111:1181-1190. doi:10.1172/JCI200316651.

18. Atkinson, J.P., and Bessler, M. 2000. Paroxysmal nocturnal hemoglobinuria. In The molecular basis of blood disease. W.B. Saunders Co. Philadelphia, Pennsylvania, USA. 564-577.

19. Richards, A., et al. 2003. Mutations in human complement regulator, membrane cofactor protein (CD46), predispose to development of familial hemolytic uremic syndrome. Proc. Natl. Acad. Sci. U. S. A. 100:12966-12971.

20. Tesser, J., et al. 2001. Safety and efficacy of the humanized anti-C5 antibody h5G1.1 in patients with rheumatoid arthritis. Arthritis Rheum. 44(Suppl.):S274. (Abstr.)

21. Petri, M. 2003. Evidence-based management of thrombosis in the antiphospholipid antibody syndrome. Curr. Rheumatol. Rep. 5:370-373.

\title{
Dominant-negative diabetes insipidus and other endocrinopathies
}

\author{
John A. Phillips III \\ Division of Medical Genetics, Vanderbilt University School of Medicine, Nashville, \\ Tennessee, USA
}

\begin{abstract}
Familial neurohypophyseal diabetes insipidus (FNDI) in humans is an autosomal dominant disorder caused by a variety of mutations in the arginine vasopressin (AVP) precursor. A new report demonstrates how heterozygosity for an AVP mutation causes FNDI (see the related article beginning on page 1697). Using an AVP knock-in mutation in mice, the study shows that FNDI is caused by retention of AVP precursors and progressive loss of AVP-producing neurons.
\end{abstract}

J. Clin. Invest. 112:1641-1643 (2003). doi:10.1172/JCI200320441.

\section{Autosomal recessive, autosomal dominant, and dominant-negative mutations}

Genes are transcribed into mRNA, which is translated into a protein product. In the case of arginine vasopressin (AVP), the protein product is synthesized as a much larger prohormone, which includes AVP, its carrier protein called neurophysin, and a glycoprotein.

The activity of different versions of genes (called alleles) and the quantitative and qualitative characteristics of their respective gene products can be correlated with the incidence and

Address correspondence to: John A. Phillips III, Division of Medical Genetics, Department of Pediatrics, Vanderbilt University School of Medicine, DD-2205 Medical Center North, Nashville, Tennessee 37232-2578, USA. Phone: (615) 322-7601; Fax: (615) 343-9951; E-mail: john.a.phillips@vanderbilt.edu. Conflict of interest: The author has declared that no conflict of interest exists.

Nonstandard abbreviations used: arginine vasopressin (AVP); autosomal recessive (AR); autosomal dominant (AD); familial neurohypophyseal diabetes insipidus (FNDI); dominant-negative (DN); oxytocin (OT); supraoptic nucleus (SON); paraventricular nucleus (PVN); growth hormone $(\mathrm{GH})$; isolated GH deficiency type II (IGHD II). severity of disease. For example, the sums of the amounts of gene products synthesized from both alleles at an autosomal locus, representing the net activity of gene expression, are shown in Figure 1. When one of two paired alleles produces sufficient protein to overcome the presence of a mutation in the second allele, homeostasis is maintained and clinical manifestations of protein deficiency do not occur. In such cases, the related disorder is characterized by an autosomal recessive (AR) mode of inheritance, and the heterozygous carrier does not manifest clinical symptoms of disease (Figure 1a, left). However, when the net activity of gene expression of two mutant alleles is not sufficient to prevent disease, such as in the case of Brattleboro rats homozygous for a single-base deletion in exon 2 of both AVP genes, diabetes insipidus with an AR mode of inheritance occurs (ref. 1; Figure $1 \mathrm{a}$, right).

In contrast, when a single normal allele does not have sufficient geneexpression activity to prevent clinical symptoms of disease, the heterozygous individual is affected and the disorder is characterized by an autosomal dominant $(\mathrm{AD})$ mode of inheritance (Figure 1b). For example, familial neurohypophyseal diabetes insipidus (FNDI) occurs in subjects heterozygous for a variety of $A V P$ gene mutations.

When a mutation in one allele prevents both it and the normal allele from producing sufficient gene product to prevent clinical symptoms, the disorder is also characterized by an $\mathrm{AD}$ mode of inheritance (Figure 1c). Note that the sum of the gene-expression activities of the mutant and normal alleles is less than that of a single normal allele, because of the effect of the dominant-negative (DN) mutation on the normal allele (Figure 1c). The DN mutation decreases expression of the normal gene in the same cell so that the sum of their gene expression does not reach the threshold needed to prevent disease.

\section{FNDI}

Both the AVP and the oxytocin (OT) prohormones are synthesized in the supraoptic nucleus (SON) and paraventricular nucleus (PVN) of the hypothalamus (2). AVP and OT are produced by separate populations of magnocellular neurons in both nuclei, packaged into neurosecretory vesicles with the neurophysins, and transported to the neurohypophysis, where they are either stored or secreted into the circulation.

In three members in three generations of a Japanese family with FNDI, Nagasaki et al. (3) found a TGC-toTGA transversion at nucleotide position 1891 that encodes a Cys67Ter change. The prematurely terminated AVP product was predicted to lack part of the neurophysin II and glycoprotein moieties. While the function of AVP is well characterized, and neurophysin II is known to act as a carrier protein, the function of the glycopro- 